Kansas State University Libraries

New Prairie Press

\title{
A STEM PROFILE MODEL CALIBRATED BY NONLINEAR MIXED- EFFECTS MODELING
}

Yuqing Yang

Shongming Huang

Shawn X. Meng

Follow this and additional works at: https://newprairiepress.org/agstatconference

Part of the Agriculture Commons, and the Applied Statistics Commons

\section{(c) (1) $\Theta(9$}

This work is licensed under a Creative Commons Attribution-Noncommercial-No Derivative Works 4.0 License.

\section{Recommended Citation}

Yang, Yuqing; Huang, Shongming; and Meng, Shawn X. (2009). "A STEM PROFILE MODEL CALIBRATED BY NONLINEAR MIXED-EFFECTS MODELING," Conference on Applied Statistics in Agriculture.

https://doi.org/10.4148/2475-7772.1082

This is brought to you for free and open access by the Conferences at New Prairie Press. It has been accepted for inclusion in Conference on Applied Statistics in Agriculture by an authorized administrator of New Prairie Press. For more information, please contact cads@k-state.edu. 
A STEM PROFILE MODEL CALIBRATED BY NONLINEAR MIXEDansas State University

\author{
Yuqing Yang, Shongming Huang, and Shawn X. Meng
}

Biometrics Unit, Forest Management Branch, Alberta Department of Sustainable Resource Development, $8^{\text {th }}$ Floor, 9920-108 Street, Edmonton, AB T5K 2M4, Canada

\begin{abstract}
A stem profile model was developed for black spruce (Picea mariana (Mill.) B.S.P.) trees in Alberta, Canada using a nonlinear mixed model approach. The model included two random parameters to capture between-subject variation and a general covariance structure to model within-subject residual autocorrelation. After evaluating various covariance structures, the 4-banded toeplitz and the spatial power structures were chosen for further evaluation. The 4banded toeplitz structure provided a better fit. The model was further evaluated using an independent data set to examine its validation accuracy. Model validation results showed that the model was able to accurately predict stem diameters at the population and subject-specific levels. Both covariance structures produced reliable model predictions, but the spatial power structure was superior to the 4-banded toeplitz structure. One to four stem diameters were used to predict random parameters and to subsequently generate subject-specific predictions. At least three stem diameters were needed to achieve better subject-specific predictions than population-average predictions.
\end{abstract}

Keywords: nonlinear mixed model, black spruce, autocorrelation.

\title{
1. Introduction
}

Repeated measurement data, obtained either from permanent sample plots measured periodically over time or from sectioned trees with multiple measurements taken along the stem of each tree, are commonly used for developing forest growth and yield models. However, such data are often correlated (Gregoire 1987). Although estimated model parameters remain unbiased, ignoring autocorrelation present in the data leads to biased variance estimation for model parameters. Therefore, any hypothesis testing and confidence interval estimation on model parameters are no longer valid (Gregoire 1987, Judge et al. 1988).

Nonlinear mixed models offer an alternative for modeling correlated data (Gregoire et al. 1995, Fang and Bailey 2001). Consisting of both fixed and random parameters, nonlinear mixed models divide data variation into between- and within-subject variations and model both explicitly. Between-subject variations are modeled through random parameters, while withinsubject variations are modeled directly using a general variance-covariance structure.

Stem profile models are commonly used in forestry for predicting stem diameters, log volumes, and tree total and merchantable volumes. A common data source for developing stem profile models is tree sectioning data, with multiple measurements made on each sample tree. Therefore, within-tree observations are likely to be correlated. However, most stem profile models were developed by linear or nonlinear ordinary least squares (Kozak 1988, Huang 1994, Sharma and Zhang 2004). Over the last ten years, several stem profile models have been developed using mixed model approaches (e.g., Garber and Maguire 2003, Younger et al. 2008). However, model predictions using tree-specific information have not been demonstrated.

The objective of this study was to develop a stem profile model for black spruce (Picea mariana (Mill.) B.S.P.) trees using a nonlinear mixed model approach. Between-tree variation in 
stem diameters was accounted for by random parameters. Various covariance structures were evaluated to model residual autocorrelation. The developed model was further evaluated for its predictive ability using an independent data set.

\section{Methods}

\subsection{The data}

Two data sets were used in this study, one for model fitting and the other for model validation. The model fitting data were a part of the tree sectioning database collected by the Alberta Government under the Phase 3 Forest Inventory Program (Alberta Environmental Protection 1988). Sample trees identified from various ecoregions, stand conditions, and ages were felled. Each felled tree was then cut at stump height ( $0.3 \mathrm{~m}$ above ground), breast height (1.3 $\mathrm{m}$ above ground), $1.5 \mathrm{~m}$ above breast height, and $2.5 \mathrm{~m}$ intervals thereafter to the top of the tree. Two diameters inside bark were measured at the lower end of each section, taken at right angles to each other. The two values were averaged to get the final diameter measure at each point. Total tree height was also recorded for each tree. An independent data set of 183 trees sectioned from the buffers of the provincial permanent sample plots (Alberta Forest Service 2000) was used for model validation. A similar tree sectioning procedure was followed. Table 1 provides summary statistics for both data sets.

\subsection{Model development}

Over the years, many model forms have been proposed for modeling tree stem profiles. Our intent was not to compare those model forms. Instead, we focused on the variable-exponent model form (Kozak 1988, Newnham 1992) used in several jurisdictions in Canada and some parts of the United States (Kozak 1988, Garber and Maguire 2003, Sharma and Zhang 2004, Younger et al. 2008). It has also been shown to work well for major Alberta tree species (Huang 1994).

The basic formulation for a variable-exponent stem profile model is:

$d=k X^{C}$

where $d$ is diameter inside bark $(\mathrm{cm})$ at stem height $h(\mathrm{~m})$ above ground; $k$ is diameter inside bark (cm) at the reference height $p ; C$ is a function of various tree and stand variables; and $X=\left(1-z^{\tau}\right) /\left(1-p^{\tau}\right)$, where $z$ is the relative height $h / H$ with $H$ being total tree height (m), and $\tau$ is a constant. Newnham (1992) fixed the reference point at breast height $(p=1.3 / H)$, and $k$ became diameter inside bark at breast height.

Kozak (2004) proposed several $\tau$ values for formulating $X: 1 / 2,1 / 3$, and $1 / 4$. In addition, various functions of stand and tree variables have been adopted over the years for the exponent $C$ (Huang 1994, Kozak 2004). After evaluating various formulations for each model component, the following model was selected as the base model for further analysis:

$$
d=a_{0} D^{a_{1}} X^{b_{0}+b_{1} z+b_{2} / \exp (D / H)+b_{3} H Q^{4}+b_{4} X^{3}}
$$

where $\mathrm{D}$ is tree diameter at breast height (1.3 $\mathrm{m}$ above ground), $X=\left(1-z^{1 / 4}\right) /\left(1-p^{1 / 4}\right)$ with $\tau=1 / 4, Q=1-z^{1 / 4}, p=1.3 / H, a_{0}, a_{1}$, and $b_{0}-b_{4}$ are model parameters to be estimated, and all other variables are as previously defined.

Model (2) is used for all trees in the population. Since parameter estimates from individual trees are likely to be different, model parameters can be divided into fixed parameters, common to all trees within the population, and random parameters specific to each individual tree. Following the nonlinear mixed model approach (Davidian and Giltinan 1995, Vonesh and Chinchilli 1997), model (2) can be expressed in a general form as:

$$
\mathbf{d}_{i}=f\left(\mathbf{x}_{i}, \boldsymbol{\beta}, \mathbf{u}_{i}\right)+\boldsymbol{\varepsilon}_{i}, \boldsymbol{\varepsilon}_{i} \sim N\left(\mathbf{0}, \mathbf{R}_{i}\right)
$$


where $\mathbf{d}_{\mathrm{i}}$ is an $\left(n_{\mathrm{i}} \times 1\right)$ vector of diameters inside bark observed on a subject tree $i ; \mathbf{x}_{\mathrm{i}}$ is an $\left(n_{\mathrm{i}} \times p\right)$ matrix of covariates; $\boldsymbol{\beta}$ is a $(p \times 1)$ vector of fixed parameters common to all trees; $\mathbf{u}_{\mathrm{i}}$ is a $(q \times 1)$ vector of random parameters unique to subject tree $i$, assumed to follow a multivariate normal distribution with mean zero and a variance-covariance matrix $\mathbf{D}$, with $\mathbf{D}$ modeled as unstructured; $\varepsilon_{\mathrm{i}}$ is an $\left(n_{\mathrm{i}} \times 1\right)$ vector of the error term; $\mathbf{R}_{\mathrm{i}}$ is an $\left(n_{\mathrm{i}} \times n_{\mathrm{i}}\right)$ positive-definite variancecovariance matrix for the error term, and $\mathbf{u}_{\mathrm{i}}$ and $\varepsilon_{\mathrm{i}}$ are assumed to be independent of each other.

An important question in mixed model analysis is which parameters should be treated as fixed and which should be treated as random. An over-parameterized random effects matrix can lead to inefficient estimation and poor estimates of the standard errors of the fixed effects, whereas an over-restrictive random effects matrix may lead to invalid and biased estimation of the mean response profile (Altham 1984). Different combinations of parameter types were evaluated based on a method recommended by Fang and Bailey (2001). Each parameter in equation (2) was given the opportunity to include a random term. The Akaike's information criterion (AIC) and Schwarz's Bayesian information criterion (BIC), defined in Littell et al. (2006), were used to compare the candidate models. The following model with two random parameters was found to be the best:

$$
d_{i j}=a_{0} D_{i}^{\left(a_{1}+u_{1 i}\right)} X_{i j}^{b_{0}+b_{1} z_{i j}+b_{2} / \exp \left(D_{i} / H_{i}\right)+\left(b_{3}+u_{2 i}\right) H_{i} Q_{i j}^{4}+b_{4} X_{i j}^{3}}+\varepsilon_{i j}
$$

where $d_{\mathrm{ij}}$ is the stem diameter for measurement $j=1, \ldots, n_{\mathrm{i}}$ of tree $i=1, \ldots, m ; u_{1 \mathrm{i}}$ and $u_{2 \mathrm{i}}$ are random parameters, and all other variables are as previously defined. For model fitting data, $m=$ 304 trees and $n_{\mathrm{i}}$ varies from 4 to 12 (Table 1 ).

Once the between-tree variation was accounted for by random parameters, the next step was to specify the within-tree variation (Davidian and Giltinan 1995, Fang and Bailey 2001). Repeated measurement data are often correlated, and residual variances may also be heterogeneous. Mixed models have the advantage of efficiently modeling within-tree autocorrelation and heteroscedasticity through the incorporation of random parameters and/or the direct modeling of the within-tree covariance structure through a general variance-covariance matrix $\mathbf{R}_{i}$ (Davidian and Giltinan 1995):

$$
\mathbf{R}_{i}=\sigma^{2} \mathbf{G}_{i}^{0.5} \boldsymbol{\Gamma}_{i} \mathbf{G}_{i}^{0.5}
$$

where $\sigma^{2}$ is a scaling factor for the error dispersion, equal to the residual variance of the model; $\mathbf{G}_{\mathrm{i}}$ is an $\left(\mathrm{n}_{i} \times \mathrm{n}_{i}\right)$ diagonal matrix describing between-tree variance structure (heteroscedasticity); and $\Gamma_{\mathrm{i}}$ is an $\left(\mathrm{n}_{i} \times \mathrm{n}_{i}\right)$ matrix describing the within-tree error correlation structure.

Preliminary analyses showed no clear evidence of heteroscedasticity for eq. (4) and $\mathbf{G}_{i}$ became an identity matrix that was subsequently removed from eq. (5).

To examine residual autocorrelation, normalized residuals were evaluated (Pinheiro and Bates 2000):

$$
\mathbf{r}_{i}=\hat{\sigma}^{-1}\left(\hat{\boldsymbol{\Gamma}}_{i}^{-1 / 2}\right)^{T}\left(\mathbf{d}_{i}-\hat{\mathbf{d}}_{i}\right)
$$

where $\mathbf{d}_{i}$ and $\hat{\mathbf{d}}_{i}$ are the observed and predicted stem diameters, and $\hat{\sigma}^{2} \hat{\boldsymbol{\Gamma}}_{i}$ is the estimated variance-covariance matrix for the $i$ th within-tree errors, which is the matrix $\mathbf{R}_{i}$. Normalized residuals are an extension of studentized residuals. Studentized residuals are weighted by their respective variances only, while normalized residuals are weighted by both variances and covariances (Fortin et al. 2008). If the within-tree variance-covariance is properly handled, the normalized residuals should be approximately normal and independent.

Under the null condition of no correlation, a correlation coefficient has a standard error which is roughly $1 / \sqrt{N}$, where $N$ is the number of independent pairs of observations (Diggle et al. 2002). Correlations between normalized residuals at various lags can be calculated for each tree or for all trees combined. For this study, correlations are calculated for all trees combined 
due to smaller number of observations per tree (Table 1). The confidence interval at any level of significance can subsequently be calculated using the standard error and the $t$-value corresponding to the sample size. For this analysis, $99 \%$ confidence intervals were used to examine the significance of correlations between normalized residuals of various lags apart.

For the nonlinear least squares fit of eq. (2), correlations between normalized residuals were significantly different from zero for measurements one to seven lags apart, but not for measurements further apart. After the two random parameters were incorporated, only correlations between normalized residuals for the first three lags were still significantly different from zero. To remove the remaining within-tree autocorrelation, both serial correlation structures and spatial correlation structures were evaluated for the correlation matrix $\Gamma_{i}$. The evaluated serial correlation structures included the first-order autoregressive $\operatorname{AR}(1)$, the autoregressive moving average $\operatorname{ARMA}(1,1)$, and the banded toeplitz structures with different bands. These structures, however, are designed for analyzing equally-spaced data (Littell et al. 2006). The tree sectioning data used here were only roughly equally spaced with most sections being $2.5 \mathrm{~m}$ long. The bottom two sections were 1.0 and $1.5 \mathrm{~m}$ long, and the top section was less than $2.5 \mathrm{~m}$ in most cases. To make sure a proper covariance structure was selected, six spatial covariance structures that account for unequally-spaced data, including the power, exponent, linear, linear $\log$, Gaussian, and spherical structures, were also evaluated (Littell et al. 2006, Yang and Huang 2008).

Based on the AIC and BIC statistics, the 4-banded toeplitz and the spatial power structures were found to provide the best fits for each of the two groups of correlation structures. Between the two structures, the 4-banded toeplitz structure was better than the spatial power structure.

Various methods have been proposed to estimate the parameters of nonlinear mixed models. The most common one is the maximum likelihood method, which maximizes the likelihood of the following joint probability density function (Lindstrom and Bates 1990, Pinheiro and Bates 1995):

$$
p\left(\mathbf{d}_{i}\right)=\prod_{i=1}^{m} \int p\left(\mathbf{d}_{i} \mid \mathbf{u}_{i}\right) p\left(\mathbf{u}_{i} ; \mathbf{D}\right) d \mathbf{u}_{i}
$$

where $m$ is the total number of subjects (trees), $p\left(\mathbf{d}_{i} \mid \mathbf{u}_{i}\right)$ is the conditional density of $\mathbf{d}_{i j}$ given $\mathbf{u}_{i}$, $p\left(\mathbf{u}_{i} ; \mathbf{D}\right)$ is the density of $\mathbf{u}_{i}$, and other variables are as previously defined.

The integral in eq. (7) does not have a closed-form expression in general since the random parameters enter the model nonlinearly. Therefore, it is often approximated numerically. The most commonly applied methods are based on a linear approximation of the nonlinear mixed model by a first-order Taylor series expansion. This expansion can be either at zero, the expected value of $\mathbf{u}_{i}$ (Sheiner and Beal 1980), or around an estimate; for example, the empirical best linear unbiased predictor (eblup) of the random parameters (Lindstrom and Bates 1990).

Both expansion methods produce reliable parameter estimates, but estimated values vary depending on the method used (Pinheiro and Bates 1995, Wolfinger and Lin 1997). To evaluate the impact of the two expansion methods, model (4) was fitted by both expansion methods implemented through the SAS macro NLINMIX (Littell et al. 2006).

\subsection{Model validation}

Model (4) with the 4-banded toeplitz and the spatial power covariance structures was used for making the population-average (PA) and subject-specific (SS) diameter predictions for the validation data (Table 1). At the population level, a mean response was generated using only the fixed parameters by setting the random parameters to their expected value of zero:

$$
\hat{\mathbf{d}}_{i}=f\left(\mathbf{x}_{i}, \hat{\boldsymbol{\beta}}, \mathbf{0}\right)
$$


To make subject-specific diameter predictions, random parameters $\mathbf{u}_{i}$ were predicted

first using one or more stem diameter measurements from each subject (tree). For the zero expansion method, random parameters were predicted by an approximate Bayes estimator (Vonesh and Chinchilli 1997, Trincado and Burkhart 2006):

$$
\hat{\mathbf{u}}_{i}=\hat{\mathbf{D}} \mathbf{Z}_{i}^{T}\left(\mathbf{Z}_{i} \hat{\mathbf{D}} \mathbf{Z}_{i}^{T}+\hat{\mathbf{R}}_{i}\right)^{-1}\left[\mathbf{d}_{i}-f\left(\mathbf{x}_{i}, \hat{\boldsymbol{\beta}}, \mathbf{0}\right)\right]
$$

where $\hat{\mathbf{D}}$ is the estimated variance-covariance matrix for random parameters $\mathbf{u}_{i} ; \hat{\mathbf{R}}_{i}$ is the estimated variance-covariance matrix for the error term; and $\mathbf{Z}_{\mathbf{i}}$ is the partial derivative matrix of $\mathbf{d}_{\mathrm{i}}$ with respect to random parameters $\mathbf{Z}_{i}=\partial f\left(\mathbf{x}_{i}, \boldsymbol{\beta , 0}\right) /\left.\partial \mathbf{u}_{i}\right|_{\hat{\beta}, 0}$.

For the eblup expansion method, an iterative procedure was used to predict the random parameters:

$$
\hat{\mathbf{u}}_{i}=\hat{\mathbf{D}} \mathbf{Z}_{i}^{T}\left(\mathbf{Z}_{i} \hat{\mathbf{D}} \mathbf{Z}_{i}^{T}+\hat{\mathbf{R}}_{i}\right)^{-1}\left[\mathbf{d}_{i}-f\left(\mathbf{x}_{i}, \hat{\boldsymbol{\beta}}, \hat{\mathbf{u}}_{i}\right)+\mathbf{Z}_{i} \hat{\mathbf{u}}_{i}\right]
$$

where $\hat{\mathbf{D}}, \hat{\mathbf{R}}_{i}$ and $\hat{\boldsymbol{\beta}}$ are as defined above, but $\mathbf{Z}_{i}=\partial f\left(\mathbf{x}_{i}, \boldsymbol{\beta}, \mathbf{u}_{i}\right) /\left.\partial \mathbf{u}_{i}\right|_{\hat{\boldsymbol{\beta}}, \hat{\mathbf{u}}_{i}}$.

Since $\hat{\mathbf{u}}_{i}$ appears on both sides of (10), it must be solved iteratively. The initial predictions were obtained by setting $\mathbf{u}_{i}$ at the right hand side of eq. (10) to zero. The initial predictions were then used to update random parameter predictions after updating $\mathbf{Z}_{\mathrm{i}}$ and $f\left(\mathbf{x}_{i}, \hat{\boldsymbol{\beta}}, \hat{\mathbf{u}}_{i}\right)$ using the initial predictions. The procedure was repeated until the convergence criterion was met, i.e., until the absolute differences between the two sets of predictions were smaller than a predetermined value $\left(1 \times 10^{-6}\right.$ in this study).

After the random parameters were predicted, subject-specific predictions of stem diameters were derived by eq. (11) for zero expansion (Vonesh and Chinchilli 1997) and eq. (12) for eblup expansion:

$$
\begin{aligned}
& \hat{\mathbf{d}}_{i}=f\left(\mathbf{x}_{i}, \hat{\boldsymbol{\beta}}, \mathbf{0}\right)+\mathbf{Z}_{i} \hat{\mathbf{u}}_{i} \\
& \hat{\mathbf{d}}_{i}=f\left(\mathbf{x}_{i}, \hat{\boldsymbol{\beta}}, \hat{\mathbf{u}}_{i}\right)
\end{aligned}
$$

The prediction of random parameters will vary depending on the number of stem diameter measurements available (Calama and Montero 2004). All available stem diameter measures can be used for random parameter prediction and subsequent SS diameter predictions. Instead of using all available diameter measures, we decided to use a portion of the available data to evaluate how many stem diameter measures were needed to achieve accurate stem diameter predictions at any unmeasured stem locations. Four scenarios were evaluated, where one to four stem diameters were randomly selected from each tree for random parameter prediction. Stem diameters at the remaining locations were then predicted. The procedure was repeated 100 times for each scenario to account for random selection variability, and the diameter predictions were averaged across the repetitions.

The PA and SS stem diameter predictions for the validation data were subsequently used to evaluate the predictive ability of model (4). For this study, the evaluation was focused on prediction errors using the following statistics:

$$
\begin{aligned}
& \bar{e}=\sum_{i=1}^{m} \sum_{j=1}^{n_{i}}\left(d_{i j}-\hat{d}_{i j}\right) / \sum_{i=1}^{m} n_{i} \\
& \bar{e} \%=100 \times \bar{e} / \bar{d} \\
& \delta=\bar{e}^{2}+S D^{2}
\end{aligned}
$$

where $d_{i j}$ and $\hat{d}_{i j}$ are the $j$ th observed and predicted stem diameters for tree $i(i=1,2, \ldots, m ; j=1$, $\left.2, \ldots, n_{\mathrm{i}}\right), \bar{d}$ is the arithmetic mean of the observed stem diameters, $\bar{e}$ is the overall mean 
prediction bias, $\bar{e} \%$ is percent mean prediction bias, $S D$ is the standard deviation of the prediction errors, and $\delta$ is a combination of the bias $(\bar{e})$ and precision $(S D)$ (Cochran 1977).

Eqs. (13) to (15) were for all observations combined. These statistics were termed in this paper as the overall average statistics.

To focus on subject-specific predictions, these statistics were also calculated for each subject (tree) $i$ :

$$
\begin{aligned}
& \bar{e}_{i}=\sum_{j=1}^{n_{i}}\left(d_{i j}-\hat{d}_{i j}\right) / n_{i} \\
& \bar{e}_{i} \%=100 \times \bar{e}_{i} / \bar{d}_{i} \\
& \delta_{i}=\bar{e}_{i}^{2}+S D_{i}^{2}
\end{aligned}
$$

Once those statistics were calculated for each tree, they were averaged across all trees. The resulting statistics were termed tree average statistics.

In addition to the above statistics, the percentages of improvement of SS over PA predictions were also examined for the overall average statistics and tree average statistics for each scenario based on the 100 repetitions.

\section{Results}

\subsection{Model development}

Table 2 shows the correlation coefficients between lagged normalized residuals for model (2) fitted by nonlinear least squares and model (4) by the mixed methods with and without the 4banded toeplitz and the spatial power covariance structures. It was clear that, for the nonlinear least squares fit, significant correlation was present for the first seven lags, as the correlation coefficients were much larger than the boundary values for $99 \%$ confidence intervals. These positive correlations could be partly due to between-tree variation in model parameters, which was not accommodated in model (2). This was confirmed by the mixed model fit. With two random parameters, model (4) reduced residual autocorrelation substantially. However, significant correlation was still present for the first three lags. Both covariance structures further reduced residual autocorrelation. The 4-banded toeplitz covariance structure successfully removed all residual autocorrelation. However, residual correlations remained significant for lags one, two and four for the spatial power structure. Though the correlation coefficients were close to the boundary values for lags one and four, they were nonetheless still significant. The conclusions were the same for both expansion methods. These results confirmed that the 4banded toeplitz structure was better for the data and the model evaluated.

Table 3 provides the estimated model parameters, variance components, correlation parameters, as well as the AIC and BIC statistics for model (4) fitted with the 4-banded toeplitz and the spatial power covariance structures under the two expansion methods. All parameters were highly significant with $p$-values $<0.01$. The variance components and the correlation parameters were later used for predicting random parameters and for making subject-specific predictions of stem diameters. Based on the AIC and BIC statistics, the 4-banded toeplitz structure was better than the spatial power structure for both expansion methods.

\subsection{Model validation}

Fig. 1 shows the comparison results for the overall average statistics (eqs. (13)-(15)) for stem diameters calibrated at the population and tree levels using one to four stem diameter measurements for random parameter prediction. There was a slight tendency toward overprediction on stem diameters, as indicated by the negative mean prediction biases (Fig. 1(a)) and the percent mean prediction biases (Fig. 1(b)). However, the over-predictions were very small. 
The subject-specific predictions were always better than the population-average predictions, even when only one stem diameter was used for random parameter prediction. This was expected from a properly fitted mixed model. For SS predictions, the larger the number of stem diameters used, the better the predictions. Although the 4-banded toeplitz structure provided a better fit to the modeling data, the spatial power structure led to improved model predictions based on the validation data, as indicated by the mean prediction biases (Fig. 1(a)) and the percent mean prediction biases (Fig. 1(b)). Between the two expansion methods, zero expansion was better, except for $\mathrm{SS}_{1}$ in Fig. 1(a) where the two expansion methods led to similar prediction biases for both covariance structures. This was even more so when more stem diameters were used for SS predictions. When evaluated by the combined measure $\delta$, the differences between the two covariance structures and between the two expansion methods were very small (Fig. 1(c)).

Fig. 2 shows similar comparison results, but for tree average statistics based on equations (16)-(18). The same conclusions were reached. The prediction biases were in general smaller than their counterparts measured by the overall average statistics.

Table 4 provides the percentages of improvement of subject-specific over populationaverage predictions for the overall average statistics and tree average statistics for model (4) fitted by the two covariance structures and the two expansion methods. For the combined measure $\delta$, the SS predictions were better than the PA predictions for every repetition for each covarianceexpansion combination. The percentages of improved SS over PA predictions were always 100 and were not listed in the table. For the overall average statistics, the percentages are the same for the mean prediction bias and the percent mean prediction bias since there is only one overall mean stem diameter. In general, we concluded the following. First, zero expansion led to higher percentages than eblup expansion. Second, the percentages increased steadily with the number of stem diameters used. When one stem diameter was used for random parameter predictions, the numbers ranged from 55 for the combination of spatial power structure and eblup expansion to 67 for the combination of 4-banded toeplitz structure and eblup expansion. With three stem diameters, the percentages for zero expansion were above 90 . Although on average the 4-banded toeplitz covariance structure produced less accurate model predictions, it gave similar or slightly higher percentages.

For the tree average statistics, zero expansion resulted either in higher percentages over eblup expansion or the two expansion methods had similar results. Again, the larger the number of stem diameters used, the higher the percentages. For each scenario, the percentages were higher than their counterparts based on the overall average statistics. For example, when only one stem diameter was used for predicting random parameters, the mean prediction biases ranged from 67 to 72 and the percent mean prediction biases ranged from 75 to 86, compared to 55 to 67 for the overall average statistics (Table 4).

Both the overall average statistics and the tree average statistics indicated that in most cases three or more stem diameters were needed to ensure that subject-specific predictions were better than the population-average predictions.

\section{Discussion}

Mixed models have become a powerful and popular tool for analyzing repeatedly measured data. Data variation can be easily partitioned into between- and within-subject variations and subsequently modeled by different model components. The variance-covariance structure of the within-subject errors can be decomposed into two independent components: a variance structure and a correlation structure (Pinheiro and Bates 2000).

Mixed models account for between-tree variation through random parameters. Two random parameters were found to best capture the between-subject variation for black spruce stem profile modeling. The inclusion of these two random parameters greatly improved the 
model fit. In addition, the inclusion of the random parameters reduced the residual autocorrelations substantially, as compared to the NLS model (Table 2).

However, even with the mixed model approach, the direct modeling of residual autocorrelation was still necessary since correlations between normalized residuals remained significant for the first 3 lags. Both the 4-banded toeplitz and the spatial power covariance structures further reduced residual autocorrelations. The 4-banded toeplitz structure was able to completely eliminate all residual autocorrelations. Inclusion of the spatial power covariance structure, however, did not remove all residual autocorrelations, although substantial reductions were observed. Similar results were reported by others (e.g., Fortin et al. 2008). These model fitting results favored the 4-banded toeplitz structure. However, model validation results indicated that the spatial power structure provided better predictions. Since the developed stem profile model will be used on various data sets other than the model fitting data, and because the spatial power covariance structure is more appropriate for unequally-space data, we recommend using the spatial power covariance structure for future model predictions.

Both zero and eblup expansion methods produce reliable parameter estimates. However, several authors argued that eblup expansion performed slightly better than zero expansion in some cases but at the cost of greater computing time and instability (Pinheiro and Bates 1995, Wolfinger and Lin 1997). Hartford and Davidian (2000) demonstrated that eblup expansion was very sensitive to model specification, but less so for zero expansion. Our results showed that both expansion methods led to reliable model predictions, with zero expansion outperforming eblup expansion. Since our focus was on model predictions, zero expansion was the obvious choice.

For subject-specific stem diameter predictions, we found that the larger the number of stem diameters used for predicting random parameters, the better the predictions. This was true regardless of which expansion method was used or which error covariance structure was modeled. Therefore, as many stem diameter measurements should be used as possible for making subject-specific predictions. However, even using one stem diameter measurement resulted in reliable predictions.

\section{Summary}

A variable-exponent stem profile model was developed for black spruce trees in Alberta, Canada using a nonlinear mixed model technique. Two random parameters were used to capture between-subject variation. Two covariance structures, the 4-banded toeplitz and the spatial power structures, were used to model within-subject residual autocorrelation. Model parameters were estimated by the SAS macro NLINMIX using both zero and eblup expansion methods. Model fitting results indicated that the 4-banded toeplitz structure provided a better fit to the modeling data.

An independent validation data set was used to evaluate the predictive ability of the model. It was shown that the developed stem profile model was able to produce reliable population-average and subject-specific predictions of stem diameter, with improved predictions achieved at the subject-specific level. Although the 4-banded toeplitz covariance structure fitted the modeling data better, the spatial power structure led to better model predictions. Four scenarios were evaluated for making subject-specific stem diameter predictions, where one, two, three and four stem diameters were used for predicting random parameters. To ensure better SS over PA predictions, at least 3 stem diameters were needed for predicting random parameters and for making SS predictions. The zero expansion outperformed the eblup expansion. 


\section{Acknowledgements}

This work was supported by the Alberta Government and Forest Resource Improvement Association of Alberta (the FRIAA-GYPSY project). We thank the 11 project partners for financial and/or in kind contributions. We appreciate the valuable comments and constructive suggestions from the anonymous reviewer.

\section{References}

Alberta Environmental Protection. 1988. Alberta phase 3 forest inventory: tree sectioning manual. Pub. No. T/168, Alberta Environmental Protection, Edmonton, AB, 103 pp.

Alberta Forest Service. 2000. Permanent sample plots field procedures manual. Forest Manage. Branch, Alberta Forest Service, Edmonton, Alberta, Pub. FMOPC 83-03.

Altham, P.M.E. 1984. Improving the precision of estimation by fitting a model. J. Royal Stat. Society, Series B, 46: 118-119.

Calama, R. and Montero, G. 2004. Interregional nonlinear height-diameter model with random coefficients for stone pine in Spain. Can. J. For. Res. 34: 150-163.

Cochran, W.G. 1977. Sampling techniques. 3rd ed., Wiley, New York, 428 pp.

Davidian, M. and Giltinan, D. 1995. Nonlinear models for repeated measurement data. Chapman \& Hall, New York, 359 pp.

Diggle, P.J., Heagerty, P., Liang, K.Y. and Zeger, S.L. 2002. Analysis of longitudinal data. Oxford University Press, Oxford, UK, 379 pp.

Fang, Z. and Bailey, R.L. 2001. Nonlinear mixed effects modeling for slash pine dominant height growth following intensive silvicultural treatments. For. Sci. 47: 287-300.

Fortin, M., Bédard, S., DeBlois, J. and Meunier, S. 2008. Accounting for error correlations in diameter increment modelling: a case study applied to northern hardwood stands in Quebec, Canada. Can. J. For. Res. 38: 2274-2286.

Garber, S.M. and Maguire, D.A. 2003. Modeling stem taper of three central Oregon species using nonlinear mixed effects models and autoregressive error structures. For. Ecol. Manage. 179: 507-522.

Gregoire, T.G. 1987. Generalized error structure for forestry yield models. For. Sci. 33: 423444.

Gregoire, T.G., Schabenberger, O. and Barrett, J. P. 1995. Linear modelling of irregularly spaced, unbalanced, longitudinal data from permanent plot measurements. Can. J. For. Res. 25: $137-156$.

Hartford, A. and Davidian, M. 2000. Consequences of misspecifying assumptions in nonlinear mixed effects models. Computational Statistics and Data Analysis 34: 139-164.

Huang, S. 1994. Ecologically based individual tree volume estimation for major Alberta tree species. Pub. No. T/288, Alberta Environmental Protection, Edmonton, AB, 80 pp.

Judge, G.G., Hill, R.C., Griffiths, W.E., Lütkepohl, H. and Lee, T.C. 1988. Introduction to the theory and practice of econometrics. John Wiley \& Sons, New York, 1024 pp.

Kozak, A. 1988. A variable-exponent taper equation. Can. J. For. Res. 18: 1363-1368.

Kozak, A. 2004. My last words on taper equations. For. Chron. 80: 507-515.

Lindstrom, M.J. and Bates, D.M. 1990. Nonlinear mixed effects models for repeated measures data. Biometrics 46: 673-687.

Littell, R.C., Milliken, G.A., Stroup, W.W., Wolfinger, R.D. and Schabenberber, O. 2006. SAS for mixed models. $2^{\text {nd }}$ ed., SAS Institute Inc., Cary, NC, $814 \mathrm{pp}$.

Newnham, R.M. 1992. Variable-form taper functions for four Alberta tree species. Can. J. For. Res. 22: 210-223.

Pinheiro J.C. and Bates, D.M. 1995. Approximations to the log-likelihood function in the nonlinear mixed effects model. J. Compt. Graph Stat. 4: 12-35. 
Pinheiro, J.C. and Bates, D.M. 2000. Mixed-effects models in S and S-PLUS. Springer, New Kansas StateUniversity York, $528 \mathrm{pp}$.

Sharma, M. and Zhang, S.Y. 2004. Variable-exponent taper equations for jack pine, black spruce, and balsam fir in eastern Canada. For. Ecol. Manage. 198: 39-53.

Sheiner, L. and Beal, S.L. 1980. Evaluation of methods for estimating population pharmacokinetic parameters. I. Michaelis-Mentem model: routine clinical pharmacokinetic data. J. Pharmacokinet. Biopharm. 8: 553-571.

Trincado, G. and Burkhart, H.E. 2006. A generalized approach for modeling and localizing stem profile curves. For. Sci. 52: 670-682.

Vonesh, E.F. and Chinchilli, V.M. 1997. Linear and nonlinear models for the analysis of repeated measurements. Marcel Dekker, New York, 560 pp.

Wolfinger, R.D. and Lin, X. 1997. Two Taylor-series approximation methods for nonlinear mixed models. Computational Statistics and Data Analysis 25: 465-490.

Yang, Y. and Huang, S. 2008. Modeling percent stocking changes for lodgepole pine stands in Alberta. Can. J. For. Res. 38: 1042-1052.

Younger, N., Temesgen, H. and Garber, S. 2008. Taper and volume responses to sulfur treatment in coastal Oregon Douglas fir stands. West. J. Appl. For. 23: 142-148. 
Table 1. Summary statistics for the tree sectioning data, including the mean, standard deviation (SD), and data range for breast height diameter (D), tree height $(\mathrm{H})$, and number of sections (sect) for the model fitting (304 trees) and validation data (183 trees).

\begin{tabular}{llcc}
\hline Data & Variable & Mean \pm SD & Range \\
\hline Fitting & $\mathrm{D}(\mathrm{cm})$ & $15.03 \pm 5.25$ & $2.60-35.00$ \\
& $\mathrm{H}(\mathrm{m})$ & $13.02 \pm 3.84$ & $2.95-22.70$ \\
& sect & $7.62 \pm 1.62$ & $4-12$ \\
Validation & & & \\
& $\mathrm{D}(\mathrm{cm})$ & $14.22 \pm 4.11$ & $4.60-28.90$ \\
& $\mathrm{H}(\mathrm{m})$ & $12.97 \pm 3.70$ & $5.31-21.79$ \\
& sect & $7.86 \pm 1.63$ & $5-13$ \\
\hline
\end{tabular}

Table 2. Correlation coefficients for the normalized residuals of different lags for model (2) fitted by nonlinear least squares (NLS), and for model (4) fitted with iid residuals and with the 4-banded toeplitz (toep(4)) or spatial power covariance structure. The absolute boundary value $\rho^{*}$ is for the $99 \%$ confidence interval of zero correlation for each sample size $\left(N_{m}\right)$. Bold numbers indicate a significant correlation.

\begin{tabular}{|c|c|c|c|c|c|c|c|c|c|}
\hline \multirow[t]{2}{*}{ Lag } & \multirow[t]{2}{*}{$\mathrm{N}_{\mathrm{m}}$} & \multirow[t]{2}{*}{ NLS } & \multicolumn{3}{|c|}{ zero } & \multicolumn{3}{|c|}{ eblup } & \multirow[b]{2}{*}{$\rho^{*}$} \\
\hline & & & iid & toep(4) & power & iid & toep $(4)$ & power & \\
\hline 1 & 2011 & 0.563 & 0.407 & 0.019 & 0.061 & 0.407 & 0.020 & 0.061 & 0.058 \\
\hline 2 & 1707 & 0.389 & 0.220 & 0.042 & 0.102 & 0.220 & 0.042 & 0.101 & 0.062 \\
\hline 3 & 1403 & 0.256 & 0.085 & 0.018 & 0.026 & 0.087 & 0.018 & 0.026 & 0.069 \\
\hline 4 & 1099 & 0.167 & -0.045 & -0.070 & -0.086 & -0.044 & -0.070 & -0.085 & 0.078 \\
\hline 5 & 801 & 0.184 & 0.008 & 0.045 & 0.049 & 0.007 & 0.044 & 0.047 & 0.091 \\
\hline 6 & 525 & 0.170 & 0.005 & -0.001 & -0.045 & 0.005 & -0.001 & -0.044 & 0.113 \\
\hline 7 & 295 & 0.142 & -0.007 & -0.005 & -0.038 & -0.003 & -0.003 & -0.036 & 0.150 \\
\hline 8 & 139 & 0.131 & -0.009 & 0.008 & -0.011 & -0.012 & 0.005 & -0.016 & 0.219 \\
\hline 9 & 55 & 0.013 & -0.025 & 0.038 & -0.006 & -0.027 & 0.039 & -0.002 & 0.348 \\
\hline 10 & 18 & 0.178 & 0.071 & 0.069 & 0.163 & 0.066 & 0.070 & 0.163 & 0.608 \\
\hline
\end{tabular}


Table 3. Parameter estimates for model (4) fitted with the 4-banded toeplitz (toep(4)) and the spatial power covariance structure based on the model fitting data.

\begin{tabular}{|c|c|c|c|c|c|}
\hline & \multirow[b]{2}{*}{ parameter } & \multicolumn{2}{|c|}{ zero } & \multicolumn{2}{|c|}{ eblup } \\
\hline & & toep $(4)$ & power & toep $(4)$ & power \\
\hline \multirow[t]{7}{*}{ Fixed effects } & $a_{0}$ & 0.8147 & 0.80570 & 0.81680 & 0.80890 \\
\hline & $a_{1}$ & 1.0412 & 1.04510 & 1.04030 & 1.04370 \\
\hline & $b_{0}$ & 0.3434 & 0.33910 & 0.34260 & 0.33790 \\
\hline & $b_{1}$ & 0.4236 & 0.42540 & 0.42390 & 0.42550 \\
\hline & $b_{2}$ & -0.6104 & -0.59900 & -0.60920 & -0.59660 \\
\hline & $b_{3}$ & 0.09217 & 0.09143 & 0.09030 & 0.08953 \\
\hline & $b_{4}$ & 0.01581 & 0.01651 & 0.01621 & 0.01675 \\
\hline \multirow[t]{4}{*}{ Random effects } & $\sigma_{1}^{2}$ & 0.000079 & 0.000098 & 0.000079 & 0.000098 \\
\hline & $\sigma_{2}^{2}$ & 0.007956 & 0.009102 & 0.007785 & 0.008815 \\
\hline & $\sigma_{12}$ & 0.000113 & 0.000042 & 0.000119 & 0.000055 \\
\hline & $\sigma^{2}$ & 0.232000 & 0.237900 & 0.230800 & 0.237500 \\
\hline \multirow[t]{4}{*}{ Correlation } & $\mathrm{t}_{2}$ & 0.10900 & & 0.10810 & \\
\hline & $\mathrm{t}_{3}$ & 0.05331 & & 0.05279 & \\
\hline & $\mathrm{t}_{4}$ & 0.01786 & & 0.01781 & \\
\hline & $\rho$ & & 0.6202 & & 0.6211 \\
\hline \multirow[t]{2}{*}{ Criterion } & AIC & 3391.0 & 3553.7 & 3379.9 & 3541.3 \\
\hline & $\mathrm{BIC}$ & 3443.0 & 3598.3 & 3432.0 & 3585.9 \\
\hline
\end{tabular}

Note: $\sigma^{2}$ is residual variance, $\sigma_{1}^{2}$ and $\sigma_{2}^{2}$ are the variances for $u_{1 \mathrm{i}}$ and $u_{2 \mathrm{i}}$ and $\sigma_{12}$ is covariance between $u_{1 \mathrm{i}}$ and $u_{2 \mathrm{i}}, \mathrm{t}_{2}$ to $\mathrm{t}_{4}$ are covariance parameters for toep(4) structure, and $\rho$ is correlation parameter for spatial power structure. 
Table 4. Percentages of improvement of SS over PA predictions for the overall average statistics and the tree average statistics based on model (4) fitted with the spatial power and the 4-banded toeplitz (toep(4)) covariance structures by the two expansion methods.

\begin{tabular}{|c|c|c|c|c|c|c|}
\hline \multirow{2}{*}{ Type } & \multirow{2}{*}{ Criterion } & \multirow{2}{*}{$n$} & \multicolumn{2}{|c|}{ zero } & \multicolumn{2}{|c|}{ eblup } \\
\hline & & & toep $(4)$ & power & toep $(4)$ & power \\
\hline \multirow{4}{*}{$\begin{array}{l}\text { Overall average } \\
\text { statistics }\end{array}$} & $\overline{\mathrm{e}}, \overline{\mathrm{e}} \%$ & 1 & 63 & 62 & 67 & 55 \\
\hline & & 2 & 82 & 80 & 80 & 74 \\
\hline & & 3 & 96 & 92 & 89 & 81 \\
\hline & & 4 & 100 & 98 & 97 & 90 \\
\hline \multirow{8}{*}{$\begin{array}{l}\text { Tree average } \\
\text { statistics }\end{array}$} & $\overline{\mathrm{e}}$ & 1 & 72 & 67 & 71 & 67 \\
\hline & & 2 & 87 & 83 & 85 & 83 \\
\hline & & 3 & 99 & 94 & 94 & 89 \\
\hline & & 4 & 100 & 99 & 98 & 96 \\
\hline & $\overline{\mathrm{e}} \%$ & 1 & 86 & 86 & 84 & 75 \\
\hline & & 2 & 97 & 96 & 95 & 93 \\
\hline & & 3 & 100 & 100 & 100 & 100 \\
\hline & & 4 & 100 & 100 & 99 & 100 \\
\hline
\end{tabular}

Note: all percentages for criterion $\delta$ are 100 . 
(a)

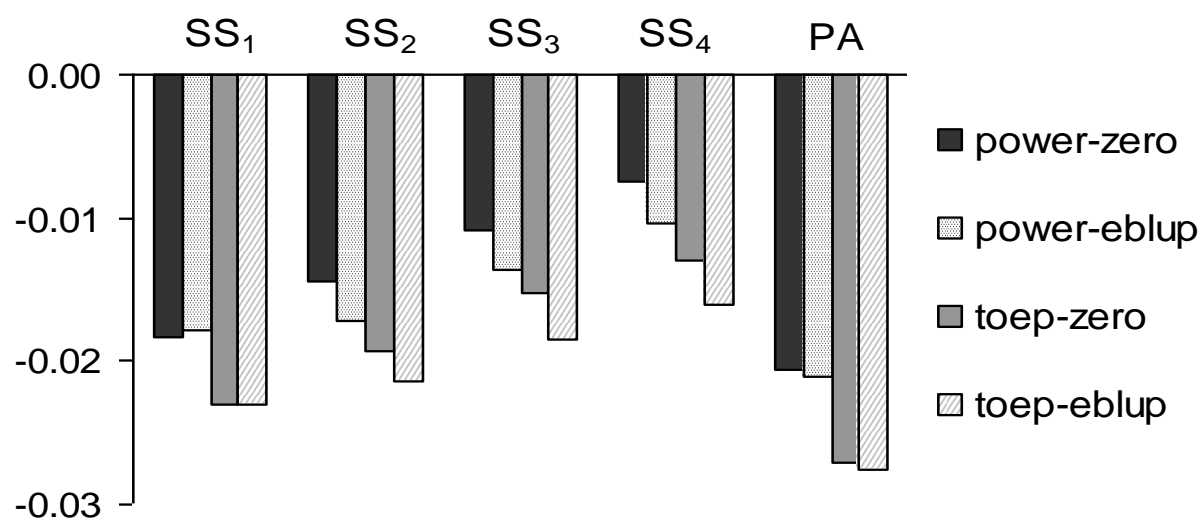

(b)
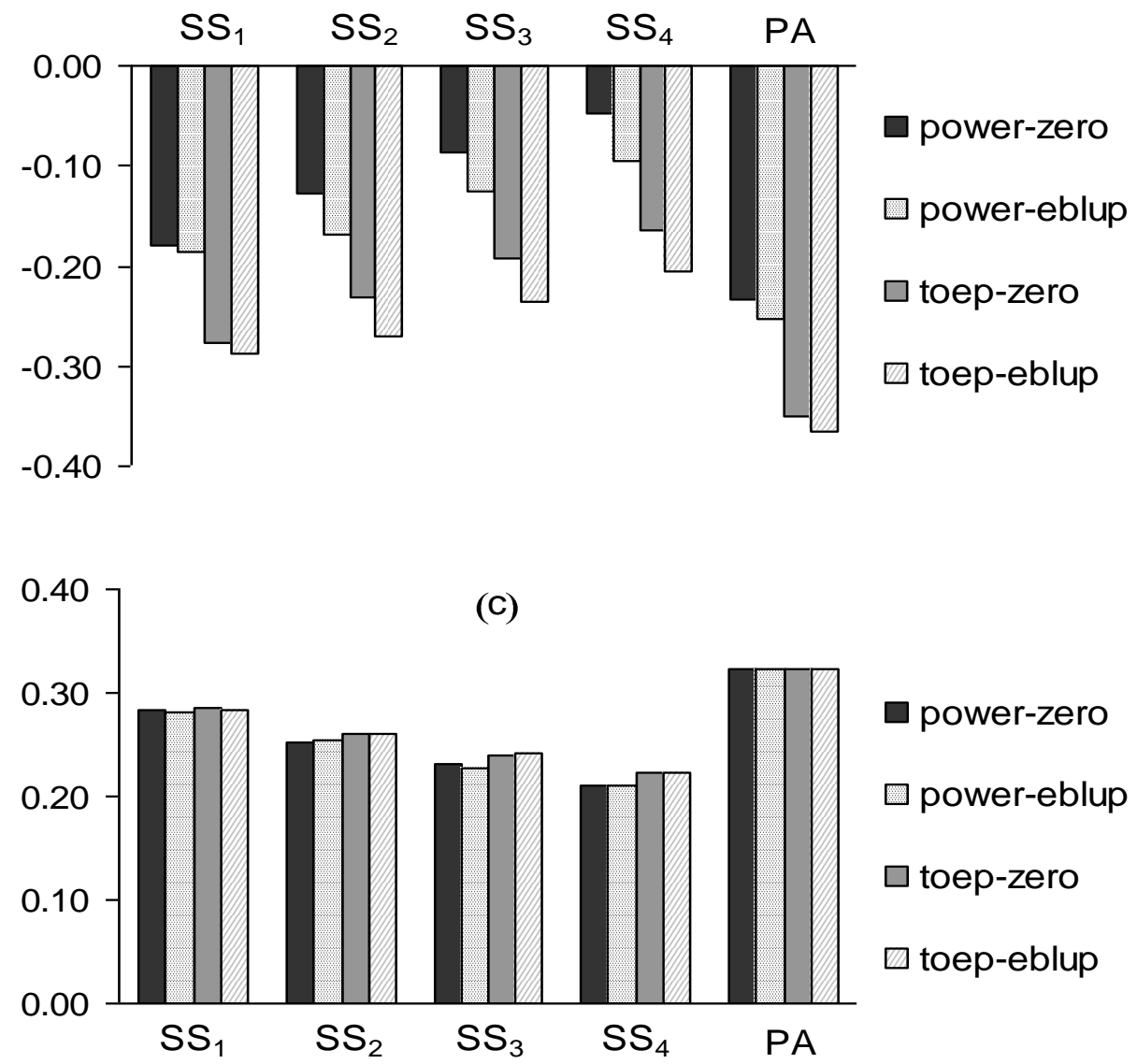

Figure 1. Comparison results for overall average statistics based on model (4) predictions at the population (PA) and tree levels where 1 to 4 stem diameters were used for random parameter prediction $\left(\mathrm{SS}_{1}\right.$ to $\left.\mathrm{SS}_{4}\right)$ : (a) the mean prediction bias, (b) the percent mean prediction bias, and (c) the combined measure $\delta$. 
(a)

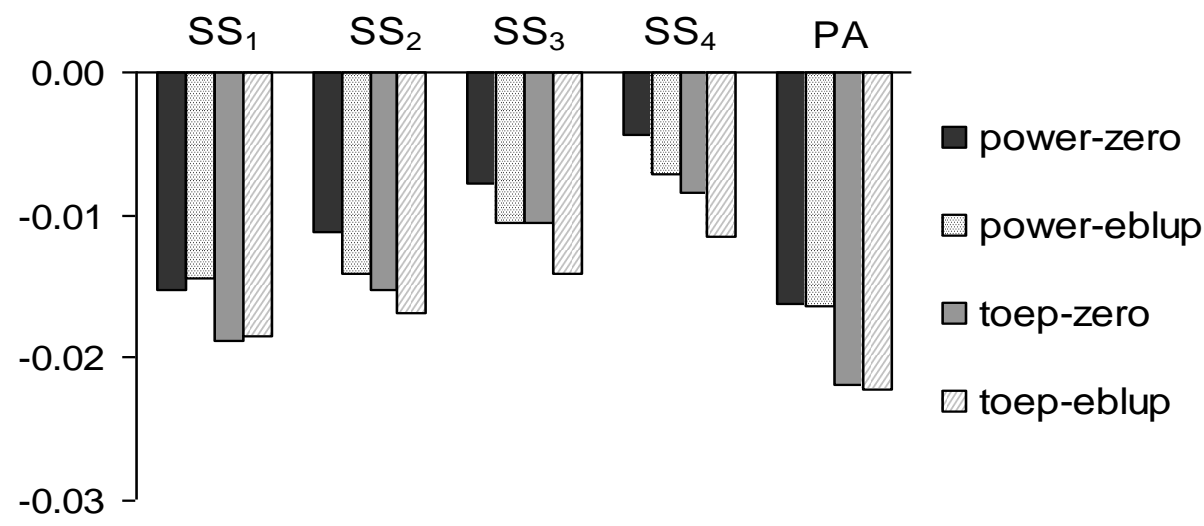

(b)
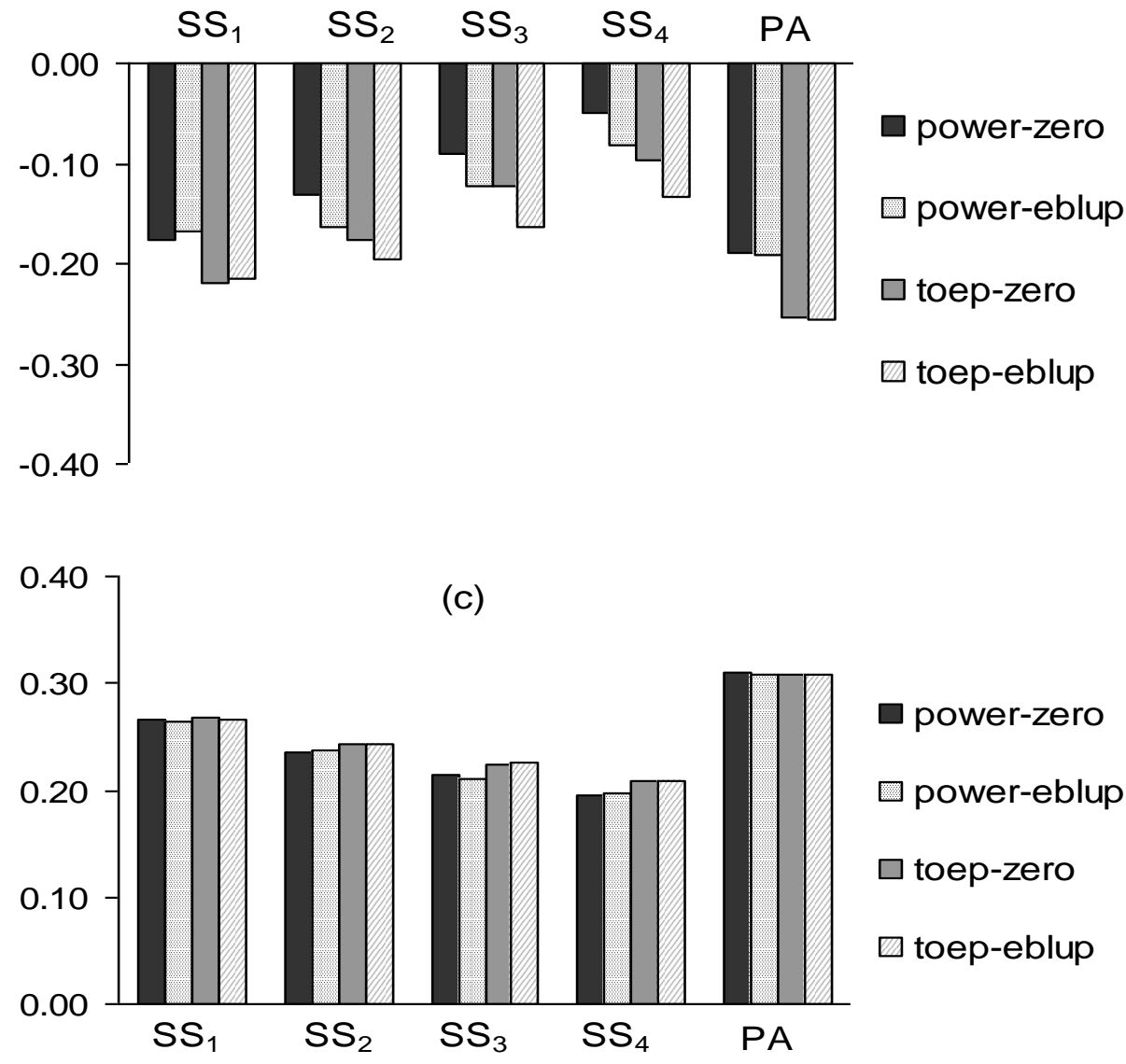

Figure 2. Comparison results for tree average statistics based on model (4) predictions at the population (PA) and the tree levels where 1 to 4 stem diameters were used for random parameter prediction $\left(\mathrm{SS}_{1}\right.$ to $\left.\mathrm{SS}_{4}\right)$ : (a) the mean prediction bias, (b) the percent mean prediction bias, and (c) the combined measure $\delta$. 